\title{
NOTE ON A CONJECTURE OF R. A. SATNOIANU
}

\author{
SHANHE WU
}

Abstract. In this paper, a conjecture posed in [R. A. Satnoianu, The proof of the conjectured inequality from the Mathematical Olympiad, Washington DC 2001, Gazeta Matematica, 106 (2001), 390-393 ] is studied once again, a generalization of Satnoianu's inequality is established.

Mathematics subject classification (2000): 26D15, 26D20, 52A40.

Keywords and phrases: Conjecture; generalization; Satnoianu's inequality; Hölder inequality; arithmetic-geometric means inequality.

\section{REFERENCES}

[1] R. A. SATNOIANU, The proof of the conjectured inequality from the Mathematical Olympiad, Washington DC 2001, Gazeta Matematica, 106 (2001), 390-393.

[2] W. Janous, On a conjecture of Razvan Satnoianu, Gazeta Matematica Seria A, XX(XCIX) (2002), $97-103$.

[3] R. A. Satnolanu, Improved GA-convexity inequalities, J. Inequal. Pure Appl. Math., 3 (5) (2002), Art. 82.

[4] S. Wu and L. Debnath, Generalizations of Aczel's inequality and Popoviciu's inequality, Indian J. Pure Appl. Math., 36 (2) (2005) 49-62.

[5] S. WU, Generalization and sharpness of power means inequality and their applications, J. Math. Anal. Appl., 312 (2005) 637-652.

[6] M. MAZUR, Problem 10944, Amer. Math. Monthly, 109 (2002), 475. 